\title{
Helium diffraction and acoustic phonons of graphene grown on copper foil
}

\author{
Amjad Al Taleb a, ${ }^{\text {, }}$ Hak Ki Yu b, c, ${ }^{1}$, Gloria Anemone a , Daniel Farías a, d, e, *, \\ Alec M. Wodtke b, c, f \\ a Departamento de Física de la Materia Condensada, Universidad Autónoma de Madrid, 28049 Madrid, Spain \\ ${ }^{\mathrm{b}}$ Institute for Physical Chemistry, University of Göttingen, 37077 Göttingen, Germany \\ ${ }^{\mathrm{c}}$ Max Planck Institute for Biophysical Chemistry, 37077 Göttingen, Germany \\ d Instituto "Nicolás Cabrera", Universidad Autónoma de Madrid, 28049 Madrid, Spain \\ e Condensed Matter Physics Center (IFIMAC), Universidad Autónoma de Madrid, 28049 Madrid, Spain \\ ${ }_{\mathrm{f}}^{\mathrm{f}}$ International Center for Advanced Studies of Energy Conversion, University of Göttingen, 37077 Göttingen, Germany
}

\section{A R T I C L E I N F O}

\section{Article history:}

Received 19 June 2015

Received in revised form

25 August 2015

Accepted 29 August 2015

Available online 3 September 2015

\section{Keywords:}

Graphene

Phonons

Helium atom scattering

CVD

\begin{abstract}
A B S T R A C T
We report helium diffraction from graphene grown by chemical vapour deposition (CVD) using copper foil. This method reveals acoustic phonons, which are physically important to thermal conductance as well as a sensitive probe of graphene's interactions with the underlying substrate. Helium diffraction is made possible by the high quality of graphene produced by a recently reported "peel-off method". The graphene lattice parameter was found to remain constant in the temperature range between 110 and $500 \mathrm{~K}$. The measured parabolic dispersion of the flexural mode along $\bar{\Gamma} \bar{M}$ allows determining the bending rigidity $k=(1.30 \pm 0.15) \mathrm{eV}$, and the graphene-Cu coupling strength $g=(5.7 \pm 0.4) \times 10^{19} \mathrm{~N} /$ $\mathrm{m}^{3}$. Unlike analytics employing atomic resolution microscopy, we obtain information on the atomic-scale quality of the graphene over mm length scales, suggesting the potential for Helium atom scattering to become an important tool for controlling the quality of industrially produced graphene.
\end{abstract}

(c) 2015 Elsevier Ltd. All rights reserved.

\section{Introduction}

Graphene's high carrier mobility [1-3] and thermal conductivity [4] promise breakthrough applications in future electronic devices [5]; however, producing mm-to cm-size samples in industrially useful amounts remains a challenge. Graphene grown by chemical vapour deposition [CVD) on copper foils is a potential solution [6-9]; unfortunately, its properties are typically degraded compared to exfoliated $\mu \mathrm{m}$-sized single crystal graphene flakes [10-13]. Degraded electrical properties may arise from chemical contamination [13], polycrystallinity, graphene interactions with its substrate [14], or reduced flatness [15]. These properties are usually examined only over length scales of $1-50 \mu \mathrm{m}$ with methods like STM, SEM and LEEM. For samples produced in industrial quantities, techniques sensitive to defect concentration, crystallinity and

\footnotetext{
* Correponding author. Departamento de Física de la Materia Condensada, Universidad Autónoma de Madrid, 28049 Madrid, Spain.

E-mail address: daniel.farias@uam.es (D. Farías).

1 These authors contributed equally to this work.
}

substrate interactions over larger length scales are urgently needed.

In this study, we report the first helium diffraction from CVD grown graphene. This method probes flatness, defect density and crystallinity over mm length scales and, furthermore, provides the material's flexural phonon spectrum, the fundamental property determining graphene's thermal conductivity [16,17] and a sensitive probe of graphene substrate interactions. We compare two graphene samples produced with modern CVD technique on two different Cu-substrates and demonstrate that a nearly ideal form of quasi-free-standing graphene can be formed on a $\mathrm{Cu}$ foil by CVD using a recently reported "peel-off" technique [18].

Helium atom scattering (HAS) is an established means of investigating the structure and dynamics of insulating as well as conducting surfaces in a completely nondestructive manner [19]. HAS diffraction provides direct information on surface crystallinity; inelastic HAS provides high-resolution $(0.5 \mathrm{meV})$ spectral information in the acoustic phonon region ( $0-50 \mathrm{meV})$ and HAS specular scattering is highly sensitivity to surface flatness and defect density [20]. This imposes strong limitations to the samples to be studied by HAS, which are usually limited to single-crystal surfaces. 
Since He beams used in HAS are typically $1 \mathrm{~mm}$ in size, the method easily provides information over a macroscopic region, which is potentially useful for nanotechnology applications like optimization of CVD graphene growth. While research aimed at improving CVD conditions for graphene is rapidly advancing; unfortunately, the HAS method's extreme sensitivity to surface defects has so far prevented detection of He diffraction from any CVD produced graphene.

In this work we show that strong HAS signals can be obtained from CVD grown graphene and that HAS data provides useful information on its quality. Specifically, we employed two new CVD methods, both of which aimed at producing improved copper catalysts [18,21,22]. In the first method, graphene was grown on an epitaxial $\mathrm{Cu}(111)$ foil using a recently developed "peel-off" scheme [18]. In the second method, thin $(\sim 100 \mathrm{~nm})$ epitaxial copper films grown on $\alpha-\mathrm{Al}_{2} \mathrm{O}_{3}(0001)$ (c-axis sapphire) are used directly as catalysts [21,22], producing graphene-Copper-Sapphire "sandwich" structures. This work shows that helium diffraction can become an important structural and morphological tool for industrially produced graphene.

\section{Experimental}

\subsection{Sample preparation}

Samples were prepared in Göttingen and then transported by courier to Madrid for HAS experiments. We have used two different $\mathrm{Cu}$ surfaces for graphene growth. In the first one, an epitaxial $\mathrm{Cu}$ (111) film (500 nm) was grown on C-plane sapphire by using electron beam evaporation method $(0.3 \mathrm{~nm} / \mathrm{s})$. The second sample was a peeled-off epitaxial $\mathrm{Cu}(111)$ foil, which was obtained by additional $\mathrm{Cu}$ electroplating and peeled-off from a sapphire substrate, as reported in our previous study [18]. The graphene growth process was different on the two $\mathrm{Cu}$ samples used. To make a highquality graphene on peeled-off epitaxial $\mathrm{Cu}(111)$ foil, we used same growth procedure as in our previous study [18]. However, the temperature should be lower than $1000^{\circ} \mathrm{C}$ to make a flat graphene on epitaxial $\mathrm{Cu}(111)$ /sapphire samples. Otherwise, due to $\mathrm{Cu}$ evaporation and migration, the surface morphology of the $\mathrm{Cu}$ film was found to be too rough for HAS. Cu migration and evaporation can be reduced at $850^{\circ} \mathrm{C}$ (see also supplementary information in Ref. [18]), but this leads to reduction of carbon solubility as well, resulting in small size of graphene. As a result, a compromise was found by preparing the graphene at $850^{\circ} \mathrm{C}$ by CVD, and doing additional ethylene annealing in UHV to get a high HAS signal. A typical growth process of graphene is as follows. First, the pressure in the growth chamber is pumped down to 3 mTorr using a mechanical pump. Second, a $40 \mathrm{sccm}$ flow of hydrogen gas is introduced into the chamber at 950 mTorr. Third, the sample was heated to $850^{\circ} \mathrm{C}$ over $50 \mathrm{~min}$. Fourth, $6 \mathrm{sccm}$ flow of methane gas with 20 sccm hydrogen is introduced into the chamber for 3 min with a total pressure of 460 mTorr for graphene synthesis; after growth, the furnace was cooled down within $1 \mathrm{~h}$ to room temperature under a $20 \mathrm{sccm}$ flow of hydrogen.

\subsection{Experimental setup}

The samples were characterized by a set of Helium Atom Scattering (HAS) and time-of-flight (TOF) measurements which were conducted in the Surface Science Laboratory in Universidad Autónoma de Madrid (LASUAM). The experiments have been carried out in two different systems, both having ultrahigh-vacuum (UHV) chambers with base pressures in the low $10^{-10} \mathrm{mbar}$ range. The first system was a He-scattering apparatus that enables determination of absolute diffraction reflectivities by measuring directly the incident beam intensity [23]. The thermal attenuation (Debye-Waller) measurements reported in the text have been performed using this system. The second chamber was a highresolution He-scattering machine with a time-of-flight arm and a fixed angle of $105.7^{\circ}$ between the incident and outgoing beam $[24,25]$. In both HAS machines, the helium beam is generated by introducing the helium gas from an 80 bar reservoir into a high vacuum chamber $\left(10^{-6}\right.$ mbar) via a $10 \mu \mathrm{m}$ platinum nozzle; the skimmer diameter is $0.6 \mathrm{~mm}$. The beam energy can be varied by changing the nozzle temperature. The beam energies used in the current work were between $17.2 \mathrm{meV}$ and $65.7 \mathrm{meV}$, with the corresponding energy spread varying from $1.5 \%$ to $5 \%$, respectively.

The samples have been mounted between a disc (back) and a ring (front) made of tantalum, and were heated by electron bombardment of the disc on the back. The temperature has been measured using a K-type thermocouple, spot-welded to the ring, touching the sample surface. At high temperatures, the sample temperature was also measured using an infrared pyrometer.

\subsection{Raman spectra}

The Raman spectra were obtained with a LabRAM HR 800 (HORIBA Yvon $\mathrm{GmbH}$ ) spectrometer under the following conditions: excitation wavelength of the laser: $\mathrm{He}-\mathrm{Ne} 633 \mathrm{~nm}$, spot size of the laser beam: $5 \mu \mathrm{m}$ in diameter, measurement time: $20 \mathrm{~s}$. The Raman spectrum of graphene grown on peeled-off epitaxial $\mathrm{Cu}(111)$ foil (Fig. 1) shows typical well-constructed monolayer graphene, namely an intensity ratio of the 2D and $G$ lines between 2 and 3 as well as a symmetric 2D band. For the case of graphene grown on epitaxial $\mathrm{Cu}(111)$ /sapphire sample, we had to reduce the growth temperature to block $\mathrm{Cu}$ evaporation during graphene growth. Although we can get the Raman signal of monolayer graphene $\left(\mathrm{CH}_{4}\right.$ source, $\left.1000^{\circ} \mathrm{C}, 10 \mathrm{~min}\right)$, the intensity is very small (compared to the graphene on peeled-off $\mathrm{Cu}$ surface) due to $\mathrm{Cu}$ evaporation. If we use ethylene $\left(\mathrm{C}_{2} \mathrm{H}_{4}\right)$ as carbon source gas for graphene growth in CVD chamber (as we used for healing of graphene in HAS study) we were able to get a clear 2D line. These results show that $\mathrm{C}_{2} \mathrm{H}_{4}$ is a compatible carbon source for graphene healing at relatively low temperatures. This phenomenon is very likely due to the relatively low carbon dissociation energy of $\mathrm{C}_{2} \mathrm{H}_{4}$ compared to $\mathrm{CH}_{4}$. The first $\mathrm{C}-\mathrm{H}$ bond energy in $\mathrm{C}_{2} \mathrm{H}_{4}$, is analogous to the first two bonds in $\mathrm{CH}_{4}$, which is expected since there is little

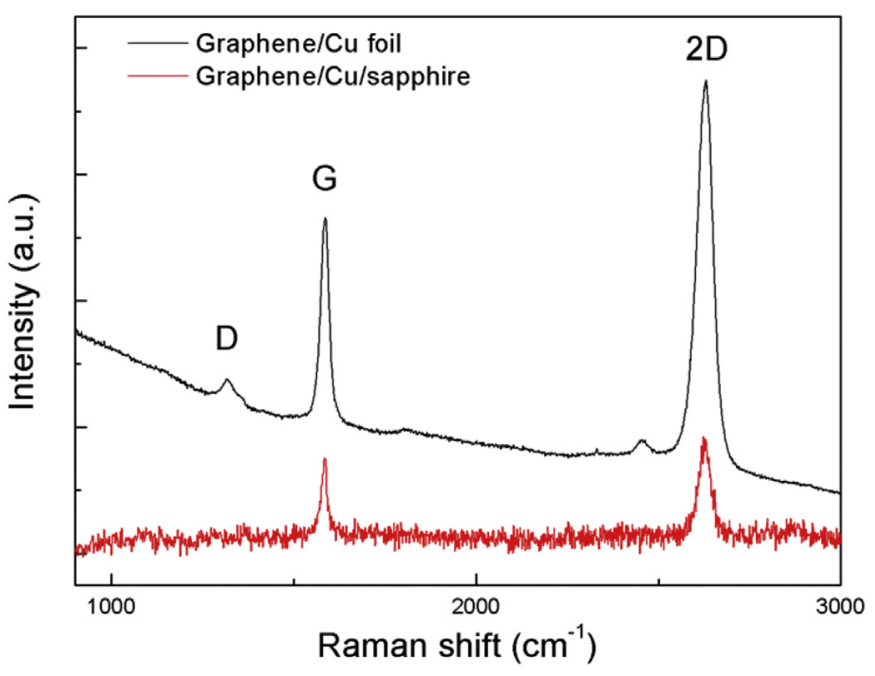

Fig. 1. Raman spectra of graphene grown with methane at $1000^{\circ} \mathrm{C}$ on the two samples used in the present work. Black curve: "Peel-off" graphene; red curve: "sandwich" graphene. (A colour version of this figure can be viewed online.) 
change in the carbon hybridization. When the second $\mathrm{H}$ is removed to form $\mathrm{C}_{2} \mathrm{H}_{2}$, the $\mathrm{C}-\mathrm{C}$ bond changes from a double to a triple bond, resulting in a very small $\mathrm{C}-\mathrm{H}$ bond energy [26].

\section{Results and discussion}

Typically, the samples delivered from Göttingen were exposed to ambient conditions for one week before being introduced into the scattering chamber for He diffraction. The as-mounted samples presented a very low He-specular reflectivity, which could be improved after heating the sample to $540 \mathrm{~K}$, presumably due to desorption of contaminants adsorbed on the surface. Further heating to $650 \mathrm{~K}$ led to a strong decrease of the specular signal to $26 \%$ of its initial value. This means that the surface quality deteriorates when heating above $650 \mathrm{~K}$. Recently it has been reported that $\mathrm{Cu}_{2} \mathrm{O}$ is formed at the graphene/Cu(111) interface during exposure to ambient conditions, and that it disappears when heating above ca. $650 \mathrm{~K}$ [27]. This is consistent with our observation of a pressure increase when heating the sample at $650 \mathrm{~K}$, which might be due to oxygen desorption. The significant decrease of $\mathrm{He}$ specular signal indicates that the density of defects on the graphene layer has increased through the heating process. The most likely interpretation is that disappearance of the $\mathrm{Cu}_{2} \mathrm{O}$ layer induces etching of the graphene.

Fig. 2 shows several angular distributions of He atoms scattered from graphene "sandwich" structures. Little or no HAS signal was observed from the as-prepared samples (not shown). However after brief heating to $540 \mathrm{~K}$, the black curve is obtained. Subsequent heating to $800 \mathrm{~K}$ with $2 \times 10^{-7}$ mbar of $\mathrm{C}_{2} \mathrm{H}_{4}$ led to further improved HAS (red curve). Further heating cycles to $860 \mathrm{~K}$ in the absence fo $\mathrm{C}_{2} \mathrm{H}_{4}$ led to additional improvement of the surface quality (green curve). The increased specular intensity is accompanied by the appearance of the first order $(10)$ and $(\overline{1} 0)$ diffraction peaks. The position of these peaks allows us to derive the lattice constant, $a=(2.44 \pm 0.02) \AA$, which agrees well with the value $2.45 \pm 0.04 \AA$ A obtained by STM for graphene/ $\operatorname{Ir}(111)$ [28], and $2.4612 \AA$, the periodicity of a single carbon layer in graphite [29]. These observations show through the appearance of graphene diffraction peaks that the heating cycles improve the long-range order and through the increased specular intensity, reduce the

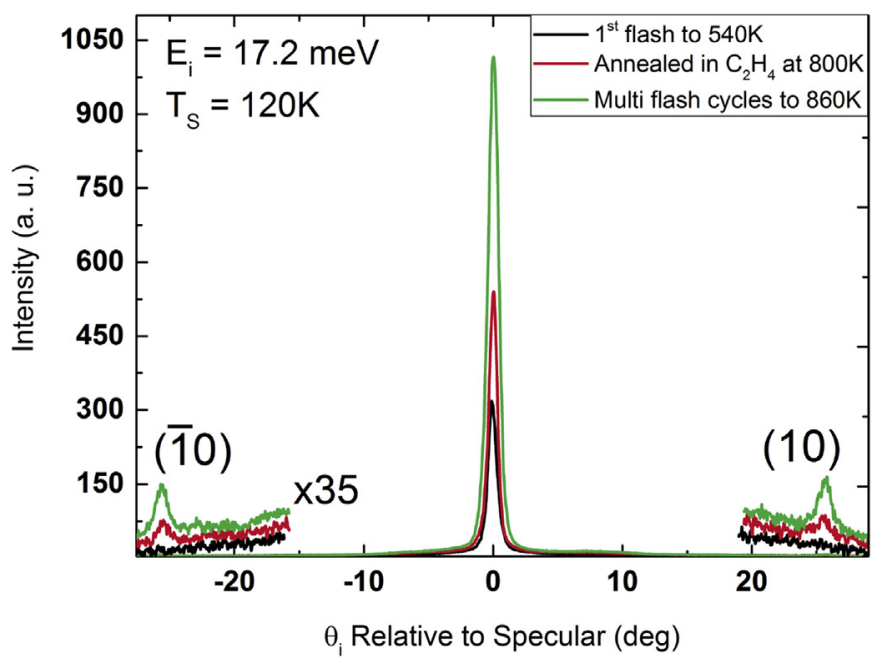

Fig. 2. He atom diffraction from CVD grown graphene on copper. Graphene prepared by "sandwich" CVD [21; 22]. Crystallinity is improved and defect density is reduced by annealing with ethylene. First order diffraction peaks reveal a lattice constant $a=(2.44 \pm 0.02) \AA$, in agreement with that of graphene. See text.(A colour version of this figure can be viewed online.) defect density resulting from, for example adsorbates or domain boundaries.

We investigated the crystallinity of the "sandwich" sample further by recording He-diffraction angular distributions for different azimuthal orientations. Fig. 3a shows three of these spectra. Each angular distribution has been measured after a small azimuthal rotation and optimization of the sample position. The black curve (denoted as $0^{\circ} \mathrm{R}$ ) shows HAS diffraction when the sample is oriented to the [11̄0] symmetry direction of the $\mathrm{Cu}(111)$ substrate, as determined independently by LEED (not shown). Here, we observe the strongest diffraction signals, denoted (10) and ( 10$)$. As before, the diffraction features yield a lattice constant that unambiguously belongs to graphene. The red curve was obtained for a sample azimuthally rotated by $30^{\circ}$ (denoted as $30^{\circ} \mathrm{R}$ ). It exhibits weaker but still clear diffraction peaks. For azimuthal orientations recorded between these two main symmetry directions, angular distributions were similar to the green curve, where diffraction is not seen.

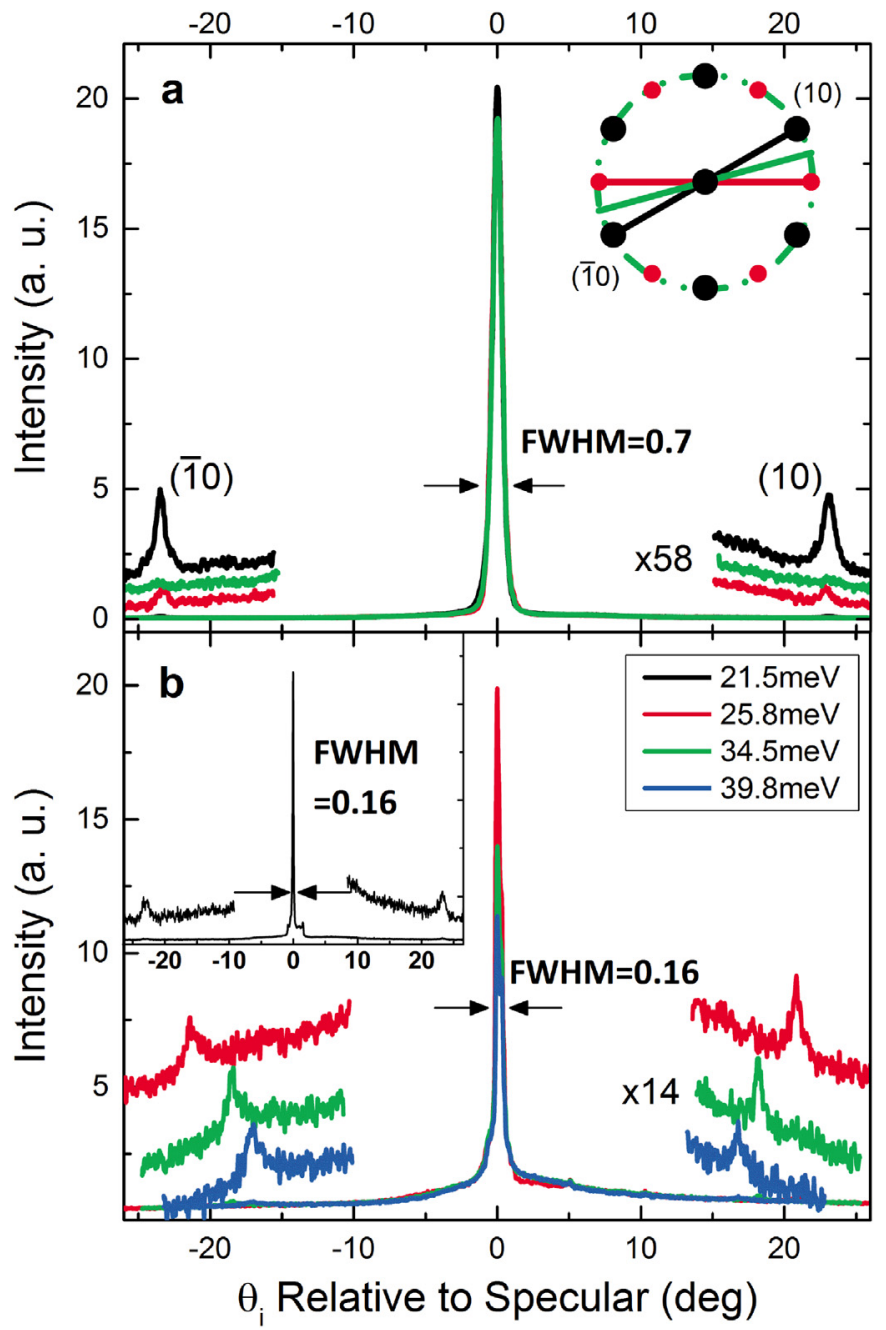

Fig. 3. Comparison of He atom scattering from two CVD grown graphene samples. (a) ("sandwich" graphene) Diffraction is seen for two azimuthal orientations (black and red). Between these azimuthal angles diffraction is undetectable (green curve). The corresponding directions on the reciprocal lattice are schematically shown in the inset. (b) ("peel-off" graphene) Diffraction is only observed for a single azimuthal orientation. These results show that two orientational domains are present for "sandwich" graphene, whereas only a single orientational domain is present for "peel-off" graphene. Inset: He-diffraction spectrum measured from a different "peel-off" graphene sample. (A colour version of this figure can be viewed online.) 
These observations give simple and valuable information about the orientation domains in this sample over a $1 \mathrm{~mm}$ area. Specifically, it shows the sample is dominated by two orientation domains $-0^{\circ} \mathrm{R}$ and $30^{\circ} \mathrm{R}-$ which are themselves oriented with respect to the $\mathrm{Cu}(111)$ substrate - that is, for the $0^{\circ} \mathrm{R}$ domain the [1120] symmetry direction of the graphene layer is parallel to the [110] direction of the $\mathrm{Cu}(111)$ lattice. The relative abundance of these two domains can also be determined from the data $\left(\sim 85 \% 00^{\circ} \mathrm{R}\right.$ and $\sim 15 \% 30^{\circ} \mathrm{R}$ ) since to first order, the intensity of the diffraction peaks is proportional to the domain's population in the $1 \mathrm{~mm}$ area probed by the experiment.

We also performed HAS experiments on "peel-off" graphene samples - representative data is shown in Fig. 3b. As before, only weak HAS signals were seen using the as prepared samples. However in contrast to the "sandwich samples", strong specular scattering as well as diffraction signals were obtained after only simple heating (to $700 \mathrm{~K}$ ); heating in ethylene was unnecessary and furthermore, had no influence on the HAS results. This shows that "sandwich samples", when probed over a 1-mm area, require significant additional $\mathrm{C}-\mathrm{C}$ bond formation to repair defects produced in the CVD. For CVD performed with the "peel-off" approach, this is not the case. We attribute this to the higher thermal stability of the several $\mu \mathrm{m}$ thick "peel-off" copper catalyst compared to the thin $(100 \mathrm{~nm})$ copper film used in the "sandwich" approach. Similar results have been obtained for several samples (the inset shows another example, taken at $\mathrm{E}_{\mathrm{i}}=21.5 \mathrm{meV}$ ). The small peak near the specular one, on the right side, corresponds very likely to specular reflection from a tilted domain on the graphene layer (since the $\mathrm{Gr}$ / $\mathrm{Cu}$-foil is very thin, not all domains lie flat on the same macroscopic plane after mounting the sample). The high specular reflectivity observed suggests that the "peel-off" graphene samples are excellent candidates to be used as focussing mirror in scanning He atom microscopy [30-33].

HAS also provides insights into the relative domain size and orientation for "peel-off" compared to "sandwich" samples. For "peel-off" samples, He diffraction was only observed for azimuthal

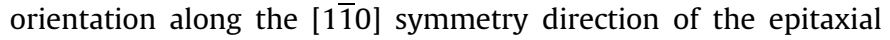
$\mathrm{Cu}(111)$ foil (again determined by LEED). This shows that exclusively $0^{\circ} \mathrm{R}$ graphene orientational domains are produced in "peeloff" CVD. In addition, the HAS results allow us to compare the average domain sizes produced by the two CVD methods, since the domain size influences the width of the specular scattering peak. By performing Gaussian fits to the specular peaks, we obtained peak widths for "peel-off" (FWHM $=0.16^{\circ}$ - instrument limited) and "sandwich" (FWHM $\left.=0.70^{\circ}\right)$ graphene. This shows that the graphene domain sizes produced by "peel-off" CVD are much larger than those from "sandwich" CVD. Since the width observed for "peel-off" graphene is limited by the resolution of our system, we can conclude that the average domain size is at least $200 \mathrm{~nm}$, i.e. one order of magnitude larger than the transfer width of our system.

HAS also allows us to investigate the interactions of graphene with the copper substrate. Fig. 4a shows HAS angular distributions as a function of sample temperature from 100 to $500 \mathrm{~K}$ using "sandwich" graphene. These data have been analysed following the same procedure reported in Ref. [34] for the LiF(001) surface. The sample temperature was stabilized for each measurement before recording the spectra within $\pm 2 \mathrm{~K}$. The diffraction peaks of graphene were detected only up to $500 \mathrm{~K}$, due to the strong attenuation caused by the Debye-Waller effect. The dependence of the diffraction angles on the lattice constant given by Bragg condition yields that a change in the lattice constant from $2.440 \AA$ to $2.445 \AA$ should produce a change in the diffraction angle from $24.80^{\circ}$ to $24.75^{\circ}$, which should be clearly detectable with our setup.

The derived graphene lattice constants are plotted in Fig. 4c over
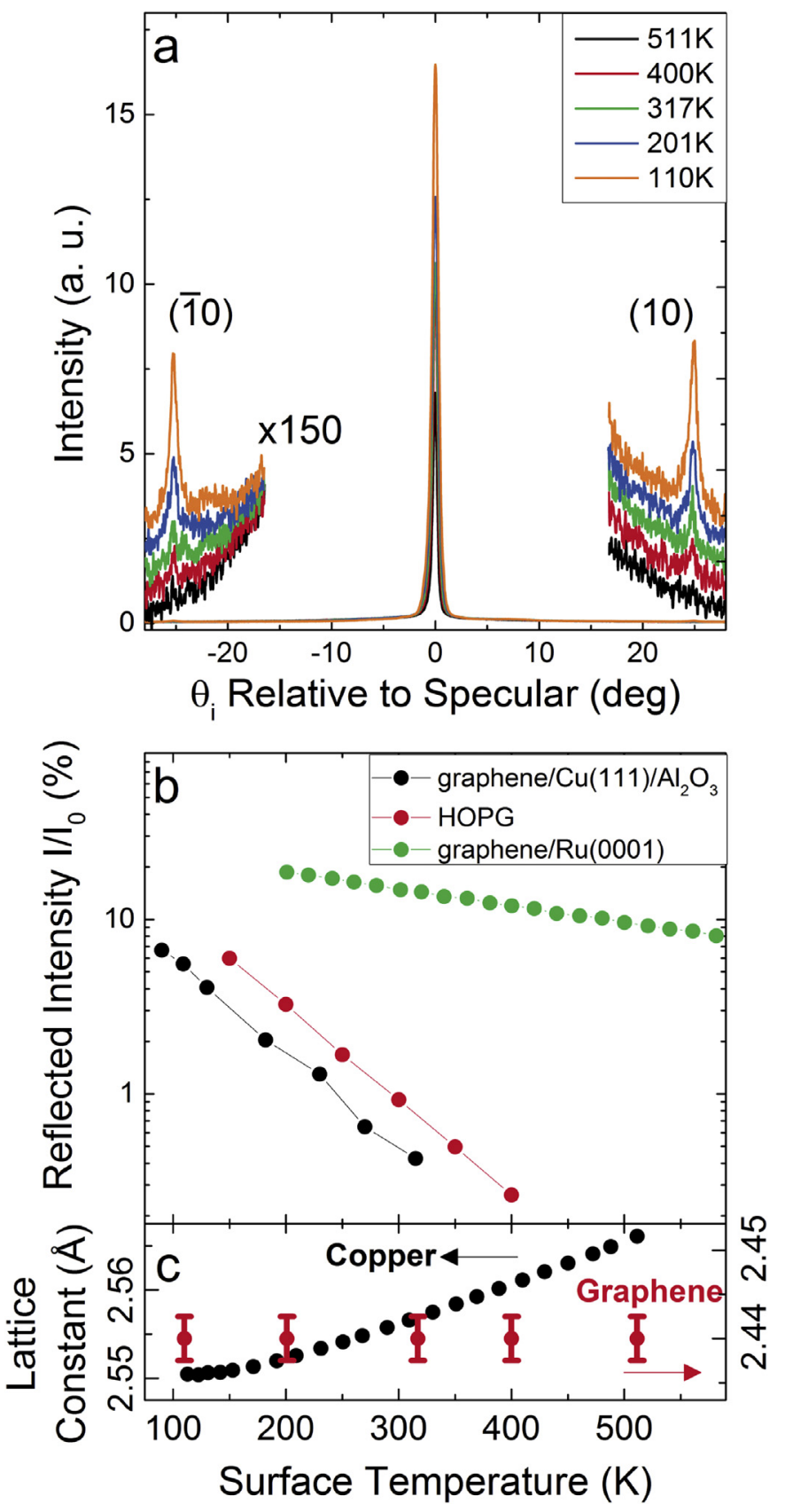

Fig. 4. (a) Temperature dependence of diffraction from "sandwich" graphene $\left(E_{i}=18 \mathrm{meV}\right)$. The graphene lattice constant derived from these data is plotted in panel (c) together with the expected variation of the $\mathrm{Cu}$ lattice constant in the same temperature range. (b) Thermal attenuation of specular He scattering for "sandwich" graphene (black); highly ordered pyrolytic graphite (data from (37)) (red) and graphene grown on $\mathrm{Ru}(0001)$ from (38) (green). The slope of the thermal attenuation curves yield the effective mass of the solid experienced by the He atom and is a sensitive probe of the strength of the interactions between the graphene and the substrate. See text. In this scale, the error bars lie within the size of the data points plotted. (A colour version of this figure can be viewed online.)

this temperature range, together with the calculated temperature dependent lattice constant of $\mathrm{Cu}(111)$ [35]. Within experimental error $(0.005 \AA)$, the derived graphene lattice constants are temperature independent, a result that is in quantitative agreement with calculations for free standing graphene [36]. This, together with the observation of a temperature independent graphene lattice constant in contact with a thermally expanding copper substrate is striking evidence of the weak interactions between copper 
and graphene.

Thermal attenuation of He specular scattering is another means to probe graphene interactions with the copper substrate, since the attenuation slope is inversely proportional to the effective mass of the solid experienced by the $\mathrm{He}$ atom [19]. Fig. 4b shows the thermal attenuation of He specular reflectivity as a function of surface temperature for three different surfaces: "sandwich" graphene, highly ordered pyrolytic graphite (HOPG) [37] and graphene grown on $\mathrm{Ru}(0001)$ [38]. Graphene is strongly bound to $\mathrm{Ru}$ by $\mathrm{C}-\mathrm{Ru}$ chemical bonds; hence, the effective mass of the solid experienced by the colliding He atom is high, leading to a small thermal attenuation slope. For both "sandwich" graphene and HOPG the thermal attenuation slope is much larger, indicating a smaller effective mass. This shows that the graphene- $\mathrm{Cu}(111)$ interaction is similarly weak to that between HOPG layers.

Measurements of thermal expansion and thermal specular attenuation give semi-quantitative information about graphene's interactions with its substrate. HAS experiments also directly reveal acoustic phonons from scattering angle resolved time-of-flight (TOF) data. As we will show, this is a valuable probe of graphene/ substrate interactions - for example it provides a contact point with first principles theory. In addition, obtaining acoustic phonons by HAS also has the potential to provide critical information on thermal properties of graphene samples as acoustic phonons strongly influence the room temperature thermal conductivity of graphene [39].

Several series of time-of-flight (TOF) spectra have been taken under different incident conditions, in order to get the phonon dispersion curves. A representative series of TOF data is shown in Fig. 5. The spectra have been converted by means of the corresponding scan curve [40] into an energy transfer scale, taking the diffuse elastic peak to set the zero energy transfer. The low intensity of the diffuse elastic peak as compared to the specular one and its sharpness (FWHM $=0.66 \mathrm{meV}$ at $E_{i}=32 \mathrm{meV}$ ) denote the good quality of the graphene layers [41]. The beam energy and surface temperature were varied to find the optimum resolution of the inelastic peaks. For peaks corresponding to low energy and momentum transfer (which correspond to inelastic peaks appearing close to the specular peak), we used low beam energy and surface temperature. Best resolution for inelastic peaks appearing at higher energy and momentum transfer was obtained keeping the beam

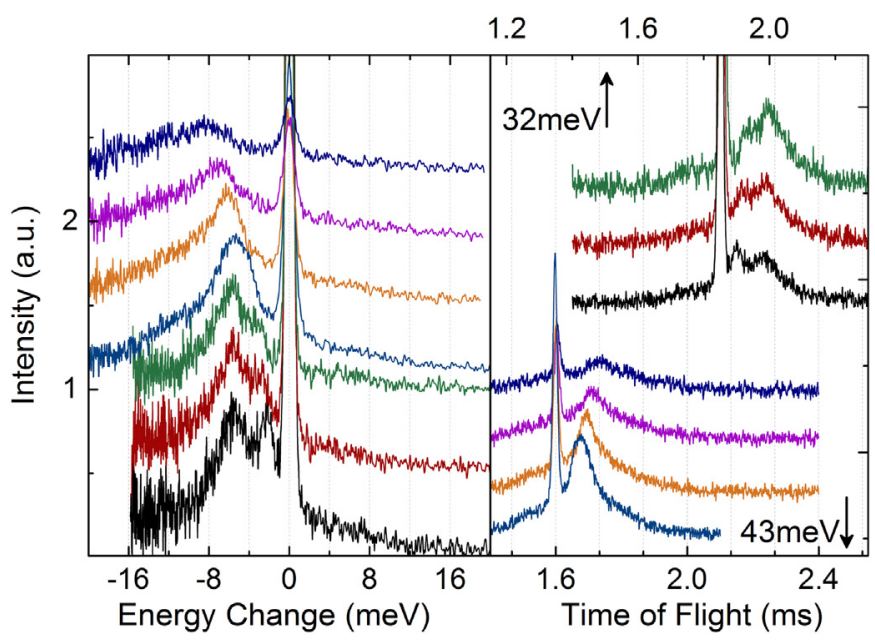

Fig. 5. Right: Time-of-flight spectra of He scattered from graphene $/ \mathrm{Cu}(111) / \mathrm{Al}_{2} \mathrm{O}_{3}$ for two different incident energies ( $43 \mathrm{meV}$ and $32 \mathrm{meV}$ ). The corresponding inelastic scattering intensity as a function of energy loss is shown on the left panel. Surface temperature is $120 \mathrm{~K}$.(A colour version of this figure can be viewed online.)
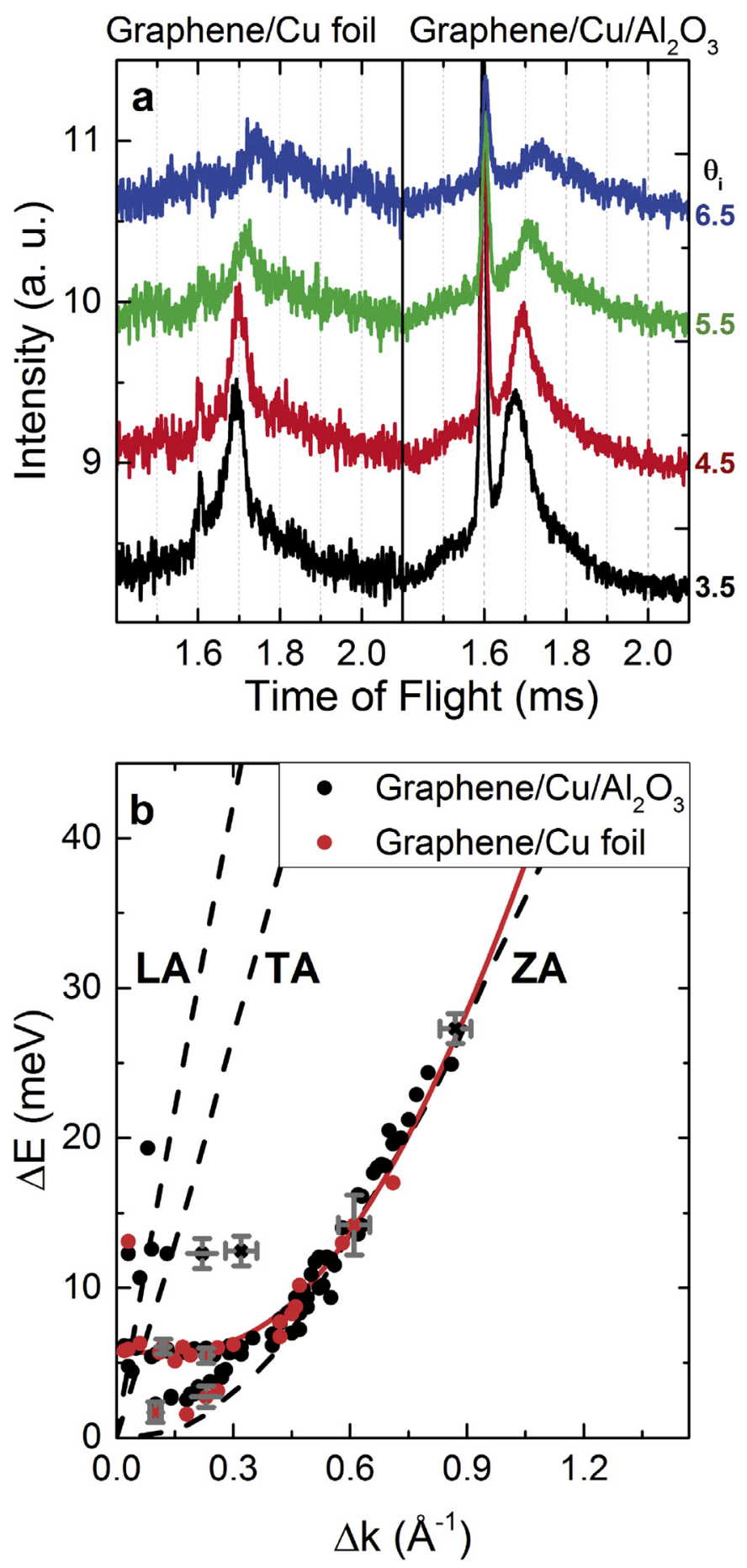

Fig. 6. (a) Angle resolved TOF spectra for "peel-off" (left) and "sandwich" (right) graphene, measured at the same conditions $\left(E_{i}=43 \mathrm{meV}, \mathrm{T}_{\mathrm{s}}=120 \mathrm{~K}\right)$. The quasielastic peaks are seen at $1.6 \mathrm{~ms}$, while inelastic scattering occurs around $1.7 \mathrm{~ms}$. (b) Experimentally derived surface phonons for "sandwich" (black dots) and "peel-off" (red dots) graphene measured along the $\bar{\Gamma} \overline{\mathrm{M}}$ direction shown with DFT calculations for free standing graphene (43) (dashed curves). Error bars are shown for some data points. Red curve - see text. (A colour version of this figure can be viewed online.)

and surface at room temperature.

Fig. 6a shows a comparison of TOF data measured at $E_{i}=43 \mathrm{meV}$ from both "peel-off" and "sandwich" graphene. A series of TOF data is shown for selected scattering angles $\left(\theta_{i}=3.5^{\circ}-6.5^{\circ}\right)$. The peak at $1.6 \mathrm{~ms}$ (TOF independent of $\theta_{i}$ ) arises from elastic scattering; the broader feature centered near $1.7 \mathrm{~ms}$ (TOF depends on $\theta_{i}$ ) results 
from inelastic scattering where acoustic phonons have been excited. The reduced intensity and width of the elastic peak in the "peel-off" TOF compared to that of the "sandwich" reflects the dramatically lower defect density of the former compared to the latter [42], results that are consistent with the previous discussion of Fig. 3.

For inelastic features, the TOF's dependence on $\theta_{i}$ allows us to obtain the dispersion curves for specific phonons. Fig. $6 \mathrm{~b}$ shows the phonon dispersion curves for "peel-off" and "sandwich" graphene measured along the $\bar{\Gamma} \bar{M}$ direction. Phonon dispersion curves for free standing graphene calculated from first principles [43] are also shown as dashed lines. Comparison to these calculations allow us to assign most of the observed phonons as flexural phonons, more specifically perpendicular acoustic (ZA) phonon modes. Surprisingly, no data points were detected for the longitudinal acoustic (LA) and transverse acoustic (TA) modes. The latter would be anyway forbidden for planar scattering in the $\bar{\Gamma} \overline{\mathrm{M}}$ direction [44], but the LA mode should be present (in fact, it has been clearly detected on the graphite (001) surface with HAS [45]). First principles phonon calculations of a graphene copper interface predict a few meV shift of the ZA mode near the $\Gamma$ point, a direct measure of the strength of the copper/graphene interaction [46]. This shift is clearly seen at $\omega_{0} \sim 6 \mathrm{meV}$ in Fig. $6 \mathrm{~b}$ and we assign this to the graphene copper interaction for the $0^{\circ} \mathrm{R}$ orientational domain. A similar feature is seen at $\omega \sim 12 \mathrm{meV}$, which can be assigned to an overtone of $\omega_{0}$.

An unshifted dispersion curve is also observed strongly resembling the ZA mode of free-standing graphene [43]. We assign this to regions of the graphene sample where the interaction with the substrate is reduced, possibly due to nanoripples [15] or inhomogeneities in the graphene-copper spacing. This could also come from the Rayleigh wave of the underlying $\mathrm{Cu}(111)$ substrate, since at this wavevector the penetration depth of He atoms is large enough to sample it [47]. Interestingly enough, the data points for the ZA mode follow a parabolic dispersion only at phonon wave vectors near the center of the Brillouin zone. A deviation from a parabolic law is observed for energies above $10 \mathrm{meV}$; above this energy, a quasi-linear behaviour is observed, as reported by recent calculations for a bilayer graphene [48].

These dispersion curves can be used to derive more fundamental quantities of the samples like the graphene- $\mathrm{Cu}$ coupling strength, $g$, and the free standing bending rigidity [49], $\kappa$, following the model developed in Ref. [50]. For free-standing graphene, the dispersion of the acoustic perpendicular ZA mode is given by Ref. [51]:

$\omega_{Z A}^{\text {free }}(\Delta K)=\sqrt{\frac{\kappa}{\rho_{2 D}}} \Delta K^{2}$,

where $\rho=7.6 \times 10^{-8} \mathrm{~g} / \mathrm{cm}^{2}$ is the two-dimensional mass density of graphene. Coupling graphene to a substrate will introduce a gap at a frequency $\omega_{0}$ at the $\Gamma$ point, and the dispersion relation is now given by:

$\omega_{Z A}^{\text {coupled }}(\Delta K)=\sqrt{\frac{\kappa}{\rho_{2 D}} \Delta K^{4}+\omega_{0}^{2}}$,

where $\omega_{0}=\sqrt{g / \rho_{2 D}}$ and $g$ is the coupling strength between graphene and substrate [50]. Therefore, from a fit to the experimental data using Eq. (2) it is possible to determine both $g$ and $\kappa$. We obtain $g=(5.7 \pm 0.4) \times 10^{19} \mathrm{~N} / \mathrm{m}^{3}$ for the graphene-Cu interaction. This derived value is $2-3$ times smaller than that for graphene/ $\mathrm{SiO}_{2}$ interfaces [52]. We also obtain $k=1.30 \pm 0.15 \mathrm{eV}$, consistent DFT calculations that predict $\kappa=1.20-1.61 \mathrm{eV}$ [49].

Our results help to elucidate the current debate on the relative contributions of different acoustic phonon modes to the thermal conductivity of graphene [39]. It is well known that the thermal conductivity in supported graphene is lower by an order of magnitude than that in free-standing graphene. It has been suggested that this reduction is caused by leaking of ZA phonons across the graphene-support interface. However, our current data prove that ZA dispersion for graphene/Cu is very similar to the one expected for free-standing graphene. The complete absence of data points from the LA mode suggests that this mode is also involved in heat conduction in graphene.

\section{Conclusions}

The structure and the acoustic phonon modes of graphene grown by chemical vapour deposition on $\mathrm{Cu}(111) / \mathrm{Al}_{2} \mathrm{O}_{3}$ and on a peel-off epitaxial $\mathrm{Cu}(111)$ foil have been measured with helium atom scattering (HAS). The graphene lattice constant obtained from the He-diffraction spectra is $a=(2.44 \pm 0.02) \AA$, and was found to be independent of surface temperature in the range between 110 and $500 \mathrm{~K}$. The bending rigidity, $k=(1.30 \pm 0.15) \mathrm{eV}$, and the graphene- $\mathrm{Cu}$ coupling strength $g=(5.7 \pm 0.4) \times 10^{19} \mathrm{~N} / \mathrm{m}^{3}$ have been determined from the measured parabolic dispersion of the flexural ZA mode along $\bar{\Gamma} \overline{\mathrm{M}}$. As we have shown, HAS can rapidly provide useful information on the crystallinity, substrate interactions, defect density and flatness of CVD grown graphene. It gives immediate report on sample quality over a $1 \mathrm{~mm}^{2}$ area. By sample rastering this could easily be increased to $\mathrm{cm}$ or larger in an industrial setting.

\section{Acknowledgements}

We gratefully acknowledge J.P. Toennies and the Max-PlanckGesellschaft for the donation of the scattering apparatus used in our experiments. This project was sponsored by the European Union, Seventh Framework Programme: Theme NMP.2012.1.4-3 Grant no. 309672. AMW and HKY acknowledge support from the Alexander von Humboldt Foundation, the Max Planck EPFL Center for Molecular Nanoscience and Technology as well as the Deutsche Forschungsgemeinschaft under CRC 1073.

\section{References}

[1] K.I. Bolotin, K.J. Sikes, Z. Jiang, M. Klima, G. Fudenberg, J. Hone, P. Kim, H.L. Stoermer, Ultrahigh electron mobility in suspended graphene, Solid State Commun. 146 (2008) 351

[2] A.K. Geim, K.S. Novoselov, The rise of graphene, Nat. Mater. 6 (2007) 183.

[3] K.S. Novoselov, A.K. Geim, S.V. Morozov, D. Jiang, Y. Zhang, S.V. Dubonos, I.V. Grigorieva, A.A. Firsov, Electric field effect in atomically thin carbon films, Science 306 (2004) 666

[4] A.A. Balandin, S. Ghosh, W. Bao, I. Calizo, D. Teweldebrhan, M. Feng, C.N. Lau, Superior thermal conductivity of single-layer graphene, Nano Lett. 8 (3) (2008) 902.

[5] P. Avouris, Z. Chen, V. Perebeinos, Carbon-based electronics, Nat. Nanotech. 2 (2007) 605.

[6] X. Li, W. Cai, J. An, S. Kim, J. Nah, D. Yang, R. Piner, A. Velamakanni, I. Jung, E. Tutuc, S.K. Banerjee, L. Colombo, R.S. Ruoff, Large-area synthesis of highquality and uniform graphene films on copper foils, Science 324 (2009) 1312.

[7] Y. Lee, S. Bae, H. Jang, S. Jang, S.E. Zhu, S.H. Sim, Y.I. Song, B.H. Hong, J.H. Ahn, Wafer-scale synthesis and transfer of graphene films, Nano Lett. 10 (2) (2010) 490.

[8] S. Bhaviripudi, X. Jia, M.S. Dresselhaus, J. Kong, Role of kinetic factors in chemical vapor deposition synthesis of uniform large area graphene using copper catalyst, Nano Lett. 10 (10) (2010) 4128.

[9] S. Bae, et al., Roll-to-roll production of 30-inch graphene films for transparent electrodes, Nat. Nanotech. 5 (2010) 574

[10] J.D. Wood, S.W. Schmucker, A.S. Lyons, E. Pop, J.W. Lyding, Effects of polycrystalline Cu substrate on graphene growth by chemical vapor deposition, Nano Lett. 11 (11) (2011) 4547.

[11] J.M. Wofford, S. Nie, K.F. McCarty, N.C. Bartelt, O.D. Dubon, Graphene Islands on Cu Foils: the interplay between shape, orientation, and defects, Nano Lett. 10 (12) (2010) 4890.

[12] H. Ago, Y. Ogawa, M. Tsuji, S. Mizuno, H. Hibino, Catalytic growth of graphene: 
toward large-area single-crystalline graphene, J. Phys. Chem. Lett. 3 (16) (2012) 2228.

[13] R.M. Iost, F. Crespilho, L. Zuccaro, H.K. Yu, A.M. Wodtke, K. Kern, K. Balasubramanian, Enhancing the electrochemical and electronic performance of CVD-grown graphene by minimizing trace metal impurities, Chem. Electrochem. 1 (12) (2014) 2070.

[14] X. Du, I. Skachko, A. Barker, E.Y. Andrei, Approaching ballistic transport in suspended graphene, Nat. Nanotech. 3 (2008) 491.

[15] G.-X. Ni, Y. Zheng, S.K. Bae, H.R. Kim, A. Pachoud, Y.S. Kim, C.-L. Tan, D. Im, JH. Ahn, B.H. Hong, B. Öezyilmaz, Quasi-periodic nanoripples in graphene grown by chemical vapor deposition and its impact on charge transport, ACS Nano 6 (2) (2012) 1158.

[16] L. Lindsay, D.A. Broido, N. Mingo, Flexural phonons and thermal transport in graphene, Phys. Rev. B 82 (2012) 115427.

[17] J.H. Seol, I. Jo, A.L. Moore, L. Lindsay, Z.H. Aitken, M.T. Pettes, X. Li, Z. Yao, R. Huang, D. Broido, N. Mingo, R.S. Ruoff, L. Shi, Two-Dimensional Phonon Transport in Supported Graphene, Science 328 (2010) 213.

[18] H.K. Yu, K. Balasubramanian, K. Kim, J.L. Lee, M. Maiti, C. Ropers, J. Krieg, K. Kern, A.M. Wodtke, Chemical vapor deposition of graphene on a "peeledoff"epitaxial $\mathrm{Cu}(111)$ foil: a simple approach to improved properties, ACS Nano 8 (8) (2014) 8636.

[19] D. Farías, K.H. Rieder, Atomic beam diffraction from solid surfaces, Rep. Prog. Phys. 61 (1998) 1575.

[20] B. Poelsema, G. Comsa, Scattering of Thermal Energy Atoms from Disordered Surfaces, Springer, Berlin, 1989.

[21] K.M. Reddy, A.D. Gledhill, C.-H. Chen, J.M. Drexler, N.P. Padture, High quality, transferable graphene grown on single crystal $\mathrm{Cu}(111)$ thin films on basalplane sapphire, App. Phys. Lett. 98 (2011) 113117.

[22] B. Hu, et al., Epitaxial growth of large-area single-layer graphene over Cu(111)/sapphire by atmospheric pressure CVD, Carbon 50 (2012) 57.

[23] P. Nieto, D. Barredo, D. Farías, R. Miranda, In-plane and out-of-plane diffraction of $\mathrm{H}_{2}$ from $\mathrm{Ru}(001)$, J. Phys. Chem. A 115 (25) (2011) 7283.

[24] H.J. Ernst, E. Hulpke, J.P. Toennies, Helium-atom-scattering study of the structure and phonon dynamics of the w(001) surface between 200 and 1900 k, Phys. Rev. B 46 (1992) 16081.

[25] D. Barredo, G. Laurent, P. Nieto, D. Farías, R. Miranda, High-resolution elastic and rotationally inelastic diffraction of D2from NiAl(110), J. Chem. Phys. 133 (2010) 124702.

[26] C.W. Bauschlicher Jr., S.R. Langhoff, Theoretical study of the C-H bond dissociation energies of $\mathrm{CH}_{4}, \mathrm{C}_{2} \mathrm{H}_{4}, \mathrm{C}_{2} \mathrm{H}_{4}$, and $\mathrm{H}_{2} \mathrm{C}_{2} \mathrm{O}$, Chem. Phys. Lett. 177 (2) (1991) 133.

[27] S. Ogawa, et al., Graphene growth and carbon diffusion during vacuum heating on $\mathrm{Cu}(111) / \mathrm{Al}_{2} \mathrm{O}_{3}$ substrates, Jpn. J. Appl. Phys. 52 (2013) 110122.

[28] A.T. N'Diaye, J. Coraux, T.N. Plasa, C. Busse, T. Michely, Structure of epitaxial graphene on $\operatorname{Ir}(111)$, New J. Phys. 10 (2008) 043033.

[29] J.B. Nelson, D.P. Riley, The thermal expansion of graphite from 15c. to 800c.: part I, Exp. Proc. Phys. Soc. 57 (1945) 477.

[30] B. Holst, W. Allison, An atom-focusing mirror, Nature 390 (1997) 244.

[31] M. Koch, S. Rehbein, G. Schmahl, T. Reisinger, G. Bracco, W.E. Ernst, B. Holst Imaging with neutral atoms-a new matter-wave microscope, J. Microsc. 229 (2008) 1 .

[32] K. Fladischer, et al., An ellipsoidal mirror for focusing neutral atomic and molecular beams, New J. Phys. 12 (2010) 033018.
[33] D. Barredo, F. Calleja, P. Nieto, J.J. Hinarejos, G. Laurent, A.L. Vázquez de Parga, D. Farías, R. Miranda, A quantum-stabilized mirror for atoms, Adv. Mater. 20 (2008) 3492

[34] Y. Ekinci, J.P. Toennies, Thermal expansion of the LiF(001) surface, Surf. Sci. $563(2004) 127$.

[35] K.H. Chae, H.C. Lu, T. Gustafsson, Medium-energy ion-scattering study of the temperature dependence of the structure of Cu(111), Phys. Rev. B 54 (1996) 14082.

[36] K.V. Zakharchenko, M.I. Katsnelson, A. Fasolino, Finite temperature lattice properties of graphene beyond the quasiharmonic approximation, Phys. Rev. Lett. 102 (2009) 046808.

[37] J.P. Oh, T. Kondo, D. Hatake, J. Nakamura, Elastic and inelastic scattering components in the angular intensity distribution of He scattered from graphite, Surf. Sci. 603 (2009) 895.

[38] A. Politano, B. Borca, M. Minniti, J.J. Hinarejos, A.L. Vazquez de Parga, D. Farías, R. Miranda, Helium reflectivity and Debye temperature of graphene grown epitaxially on Ru(0001), Phys. Rev. B 84 (2011) 035450.

[39] D.L. Nika, A.A. Balandin, Two-dimensional phonon transport in graphene, J. Phys. Condens. Mater 24 (2012) 233203.

[40] D.M. Smilgies, J.P. Toennies, Resolution and intensity considerations of an ideal He atom time-of-flight spectrometer for measurements of surface phonon dispersion curves, Rev. Sci. Instrum. 59 (1988) 2185.

[41] F. Hofmann, J.P. Toennies, J.R. Manson, A comprehensive experimental study of the dynamical interaction of $\mathrm{He}$ atoms with $\mathrm{Cu}(001)$ surface phonons, J. Chem. Phys. 101 (1994) 10155.

[42] J.P. Toennies, Experimental Determination of Surface Phonons by Helium Atom and Electron Energy Loss Spectroscopy. Ch. 5 in Surface Phonons, Springer-Verlag, Berlin, 1991

[43] J.A. Yan, W.Y. Ruan, M.Y. Chou, Phonon dispersions and vibrational properties of monolayer, bilayer, and trilayer graphene: density-functional perturbation theory, Phys. Rev. B 77 (2008) 125401.

[44] F. de Juan, A. Politano, G. Chiarello, H.A. Fertig, Symmetries and selection rules in the measurement of the phonon spectrum of graphene and related materials, Carbon 85 (2015) 225.

[45] G. Benedek, G. Brusdeylins, C. Heimlich, J.P. Toennies, U. Valbusa, Surface phonons in graphite(001), Surf. Sci. 178 (1986) 545.

[46] L. Chen, Z. Huang, S. Kumar, Phonon transmission and thermal conductance across graphene/Cu interface, Appl. Phys. Lett. 103 (2013) 123110.

[47] I.Yu. Sklyadneva, G. Benedek, E.V. Chulkov, P.M. Echenique, R. Heid, K.P. Bohnen, J.P. Toennies, Mode-selected electron-phonon coupling in superconducting $\mathrm{Pb}$ nanofilms determined from He atom scattering, Phys. Rev. Lett. 107 (2011) 095502.

[48] A.I. Cocemasov, D.L. Nika, A.A. Balandin, Engineering of the thermodynamic properties of bilayer graphene by atomic plane rotations: the role of the outof-plane phonons, Nanoscale 7 (2015) 12851.

[49] Y. Wei, B. Wang, J. Wu, R. Yang, M.L. Dunn, Bending rigidity and Gaussian bending stiffness of single-layered graphene, Nano Lett. 13 (2013) 26.

[50] B. Amorin, F. Guinea, Flexural mode of graphene on a substrate, Phys. Rev. B 88 (2013) 115418.

[51] L.J. Karssemeijer, A. Fasolino, Phonons of graphene and graphitic materials derived from the empirical potential LCBOPII, Surf. Sci. 605 (2011) 1611.

[52] B.N.J. Persson, H. Ueba, Heat transfer between weakly coupled systems: Graphene on a-SiO 2 , Europhys. Lett. 91 (2010) 56001. 